\author{
폐동맥에서 기시된 좌측 관상동맥 및 이중 우측 관상동맥과 관련된 \\ 심근경색 \\ 전남대학교병원 순환기내과 \\ 김지은 · 김계훈·조재영·안영근 · 정명호· 조정관·박종춘
}

\title{
Myocardial Infarction Associated with Anomalous Origin of the Left Coronary Artery from the Pulmonary Artery and Double Right Coronary Artery
}

\author{
Ji Eun Kim, Kye Hun Kim, Jae Yeong Cho, Young Keun Ahn, Myung Ho Jeong, Jeong Gwan Cho, and Jong Chun Park \\ Department of Cardiovascular Medicine, Chonnam National University Hospital, Gwangju, Korea
}

Anomalous origin of the left coronary artery from the pulmonary artery (ALCAPA) is a rare coronary artery anomaly and double right coronary artery (RCA) is a very rare coronary anomaly. Because patients with ALCAPA usually die within 1 year of being born due to myocardial infarction (MI) and heart failure, ALPACA is very rarely seen in adults. Here, we report an extremely rare asymptomatic case of MI, presumably caused by ALCAPA and double RCA, and provide a review of the literature. This is the first reported case of coronary artery anomaly that had both ALCAPA and double RCA. (Korean J Med 2019;94:215-220)

Keywords: Coronary vessel anomalies; Myocardial infarction; Pulmonary artery

\section{INTRODUCTION}

Anomalous origin of the left coronary artery from the pulmonary artery (ALCAPA) is a very rare congenital coronary artery anomaly in adults because of the high mortality rate within 1 year of birth [1]. Adult ALCAPA may be asymptomatic, but it can cause myocardial infarction (MI) or sudden cardiac death [2]. Double orifice right coronary artery (RCA) is also among the rarest coronary anomalies, and it may occur in isolation or combined with other anomalies $[3,4]$. However, there has been no previous report on the combination of double RCA and ALCAPA complicated by MI. Here, we report an extremely rare asymptomatic case of MI caused by ALCAPA and double RCA, and also provide a review of the literature.

\section{CASE REPORT}

A 54-year-old male presented with an electrocardiographic (ECG) abnormality incidentally detected during a preoperative evaluation for spinal surgery. The patient had been completely

Received: 2018. 2. 2

Revised: 2018. 3. 22

Accepted: 2018. 3. 22

Correspondence to Kye Hun Kim, M.D., Ph.D.

Department of Cardiovascular Medicine, Chonnam National University Hospital, 42 Jebong-ro, Dong-gu, Gwangju 61469, Korea Tel: +82-62-220-6978, Fax: +82-62-223-3105, E-mail: christiankyehun@hanmail.net 
asymptomatic except for low back pain associated with spinal stenosis. No abnormal findings were detected on routine laboratory or cardiac enzyme tests. The ECG revealed atrial fibrillation and pathologic Q-waves in precordial leads V1-3, suggesting an old anterior wall MI.

Two-dimensional echocardiography revealed an enlarged left ventricular (LV) chamber with end-diastolic dimension of 62 $\mathrm{mm}$, as well as akinesia, and wall thinning of the mid to apical septum and anterior wall of the LV with an ejection fraction of $50 \%$. An abnormally dilated RCA was noted; color Doppler echocardiography revealed multiple abnormal color flow signals in the inter-ventricular septum and anterior wall of the LV, suggesting multiple collateral channels for the infarcted myocardium or abnormal coronary vessels (Fig. 1).

A myocardial perfusion scan was done to evaluate myocardial viability and revealed a non-viable infarction in the left anterior descending (LAD) artery territory, including the mid to apical septum and anterior wall (Fig. 2). Computed tomography (CT) coronary angiography (CAG) revealed a markedly dilated LAD artery originating from the main pulmonary artery (PA) and left circumflex coronary artery (LCx) originating from the left coronary cusp of the aorta (Fig. 3A). Two large dilated RCAs with two separate ostia originating from the right coronary cusp of the aorta were also noted (Fig. 3B). Multiple collateral channels between the RCA and LAD were detected (Fig. 3C).

Cardiac catheterization with CAG was performed to precisely evaluate the anatomy of the coronary arteries and the degree of left to right shunt. The left CAG showed only the dilated LCx arising from the left coronary cusp of the aorta (Fig. 4A), and the LAD was sequentially visualized from the distal to proximal
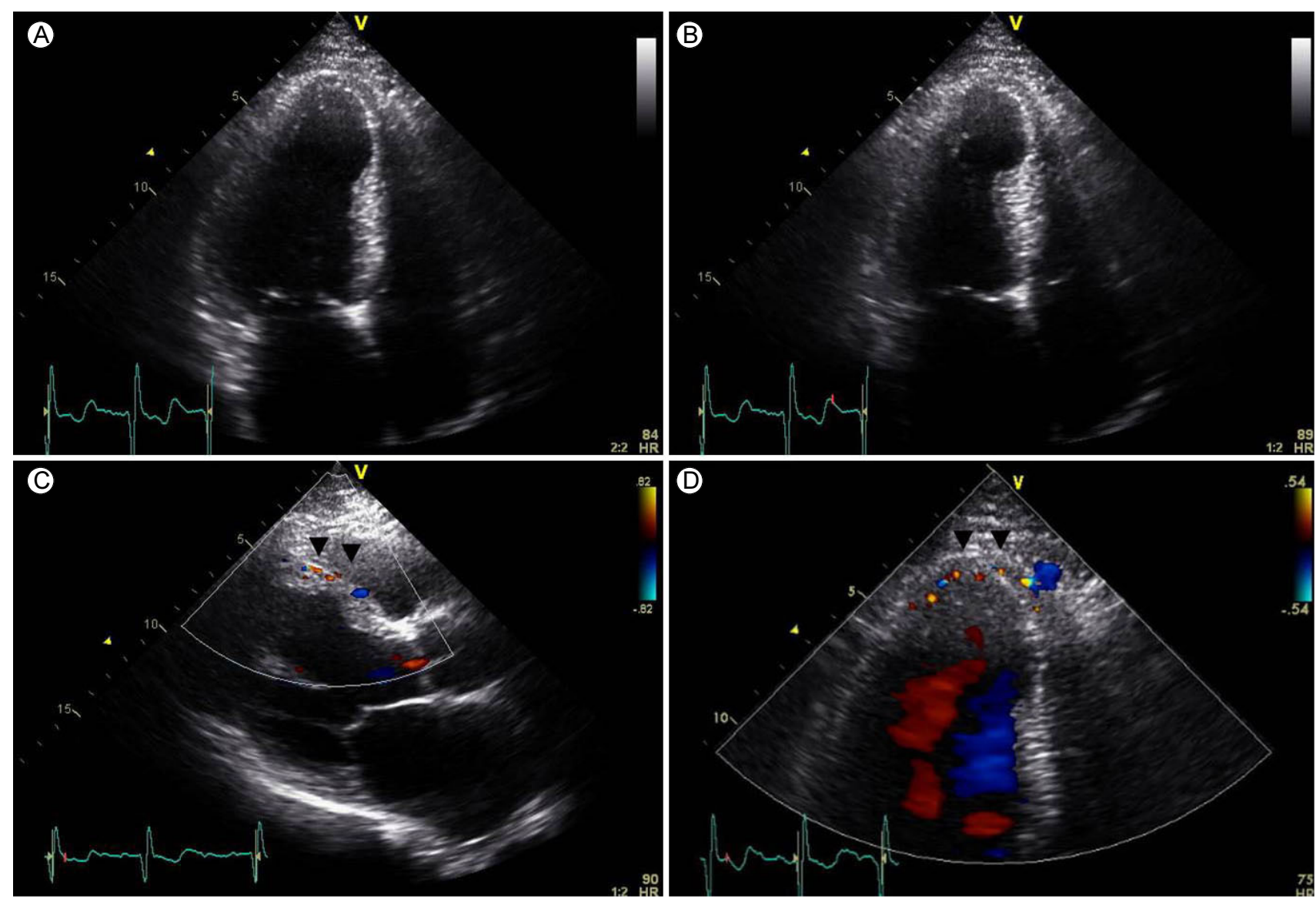

Figure 1. Echocardiography during diastole (A) and systole (B) reveals wall thinning and akinesia of the left ventricular (LV) apex and apical septum. Color Doppler imaging shows multiple turbulent color mosaics in the LV septum (C, see arrowheads) and apex (D, see arrowheads). 
- Ji Eun Kim, et al. Myocardial infarction associated with ALCAPA and double right coronary artery -

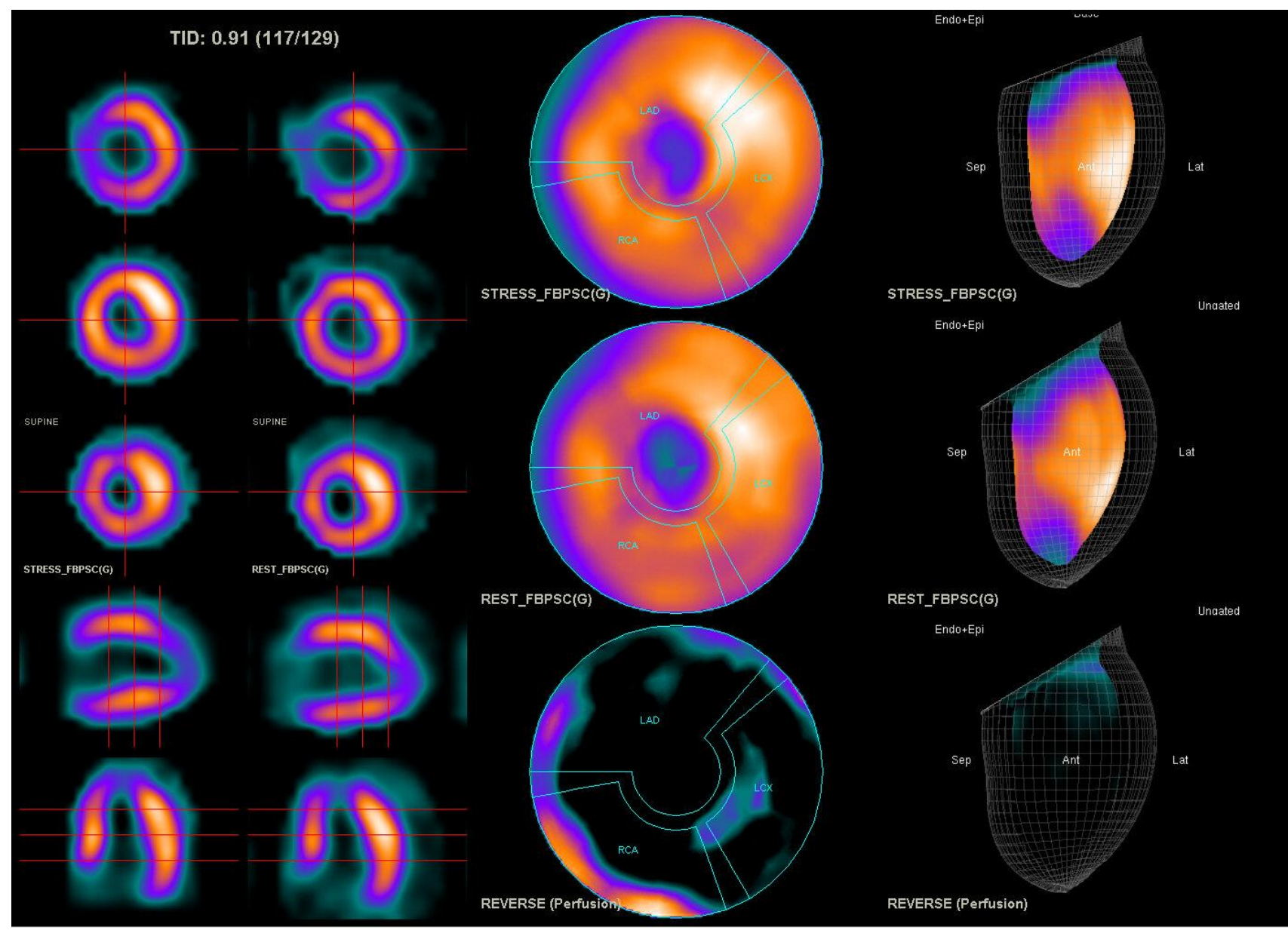

Figure 2. Myocardial perfusion scan reveals fixed perfusion defect in the mid to apical septum and anterior, indicating non-viable infarction in the left anterior descending coronary artery territory.

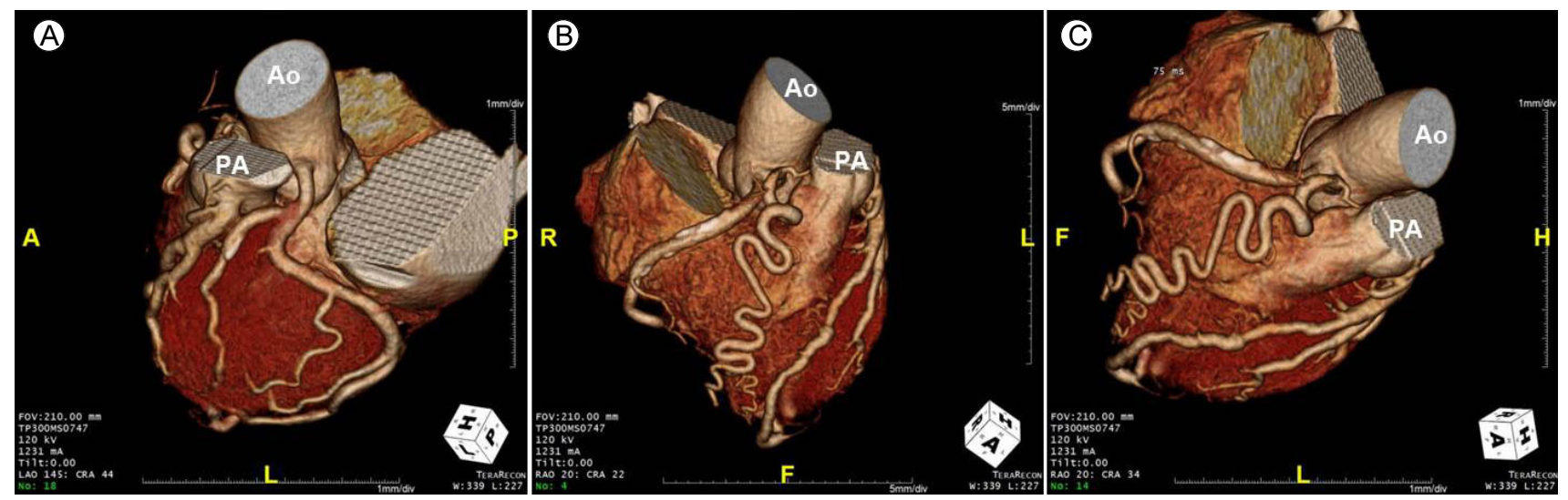

Figure 3. Computed tomography coronary angiography reveals the anomalous origin of the markedly dilated left anterior descending (LAD) coronary artery from the main pulmonary artery (PA), and left circumflex coronary artery from the left coronary cusp of the aorta (Ao) (A). Two large dilated right coronary arteries (RCAs) with the two separate ostia originating from the right coronary cusp of the aorta are shown (B). Multiple collateral channels between the RCA and LAD are also noted (C). 

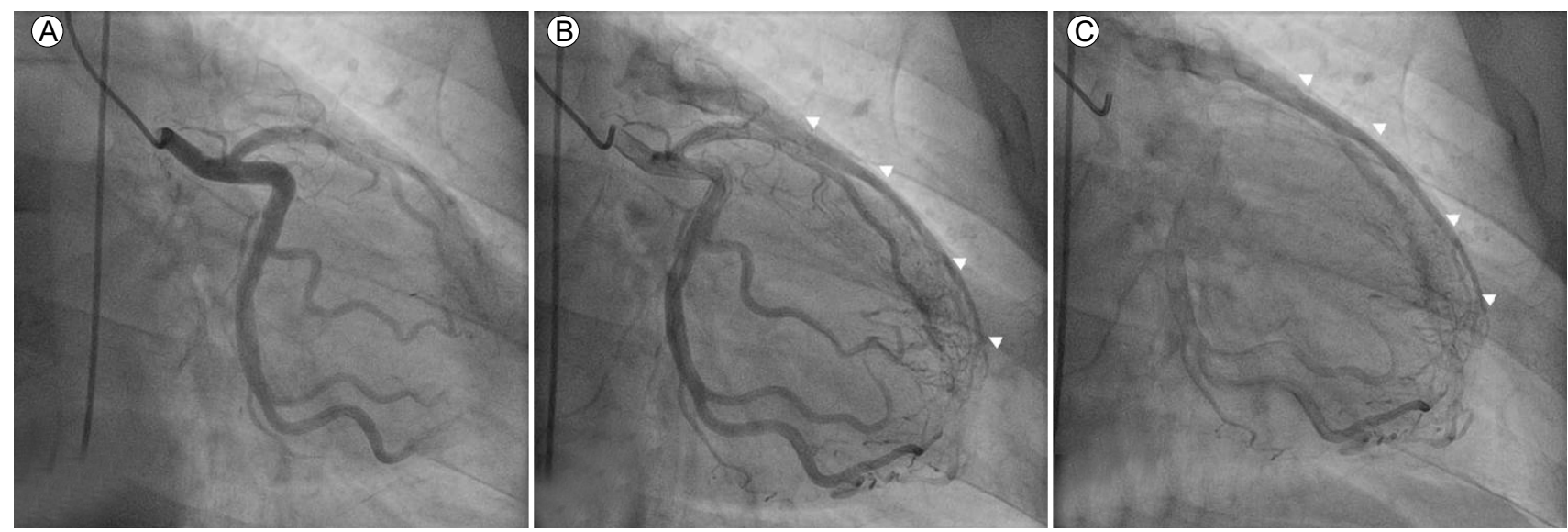

Figure 4. Left coronary angiography reveals only the dilated left circumflex coronary artery (LCx) arising from the left coronary cusp of the aorta (A), and the dilated left anterior descending (LAD) coronary artery is subsequently visualized from the distal LAD to the proximal LAD through collateral channels (Rentrop grade 2) between the LCx and LAD, before finally draining into the main PA (B and C). Arrowheads indicate LAD.
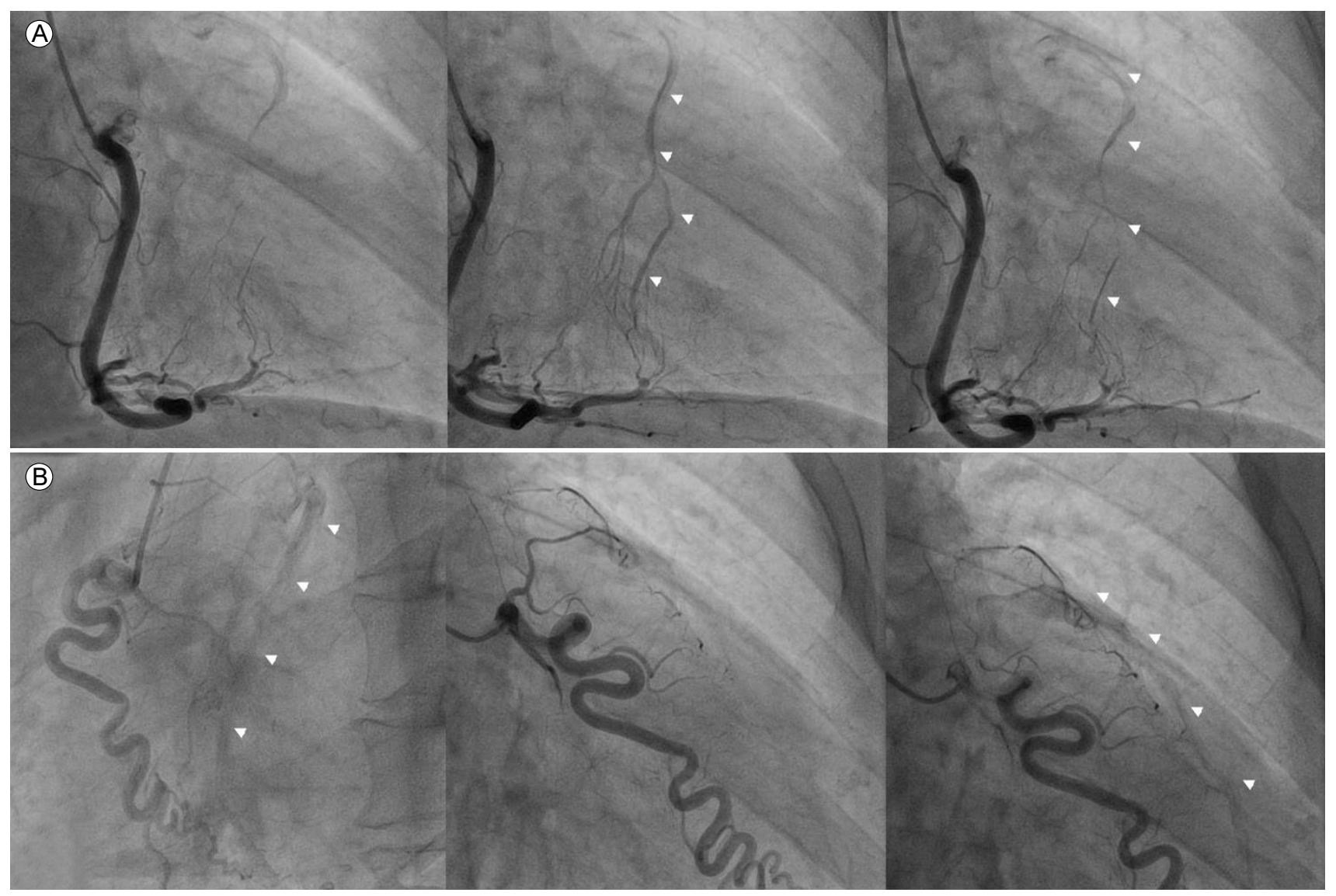

Figure 5. Right coronary angiography reveals a dilated right coronary artery (RCA) arising from the right coronary cusp of the aorta (A), and another RCA arising from a different ostium of the right coronary cusp (B). Immediately after visualizing the RCAs, the left anterior descending (LAD) coronary artery was subsequently visualized from the distal to proximal LAD through multiple collateral channels (Rentrop grade 2) between the LAD and both RCAs, before finally draining into the main PA (A and B). Arrowheads indicate LAD. 
LAD through the collateral channels from the $\mathrm{LCx}$, before finally draining into the main PA (Fig. 4B and 4C). The right CAG revealed two dilated RCAs with two separate ostia arising from the right coronary cusp of the aorta, and the LAD was sequentially visualized from the distal to proximal LAD through multiple collateral channels between the LAD and both RCAs, before finally draining into the main PA (Fig. 5). Systolic and diastolic PA pressures were normal. Oxygen set-up was noted at the level of the main PA $\left(\mathrm{O}_{2}\right.$ saturation $69.9 \%$ in the right ventricle and $78.5 \%$ in the main $\mathrm{PA}$ ), and the estimated pulmonary (Qp) to systemic flow (Qs) ratio was 1.4. Corrective surgery was recommended, but the patient opted for medical therapy, including a beta-blocker, an angiotensin-converting enzyme inhibitor, and aspirin because of lack of symptoms.

\section{DISCUSSION}

With the widespread use of multimodality cardiovascular imaging, including multi-detector CT CAG, the prevalence of coronary artery anomalies has been increasing and was reported to be $1.9-2.3 \%$ in recent studies [5,6], i.e., higher than in the era of conventional CAG [7,8]. Congenital anomalies of the coronary artery may be associated with other congenital abnormalities of the heart and/or functional abnormalities, such as myocardial ischemia, valvular disease, or heart failure, which result in increased mortality [1,7].

ALCAPA is a rare congenital coronary artery anomaly, and the proportion of patients who undergo cardiac CT with ALCAPA is about $0.05 \%$ [6]. There are many differences in clinical presentations and prognosis between pediatric and adult cases of ALCAPA. Pediatric ALCAPA usually presents with heart failure or MI, which is associated with high mortality [1]. In contrast, as in the present case, adults with ALCAPA are typically asymptomatic and have a favorable prognosis; thus, they are diagnosed incidentally during imaging studies for other purposes [1,9].

Adult ALCAPA may be associated with malignant ventricular arrhythmias and subsequent sudden cardiac death, even if the patient has been asymptomatic [1,2]; thus, close monitoring and medical therapy for myocardial ischemia or heart failure should be done, particularly for patients with MI. Regarding the pathophysiology of myocardial ischemia, as pressure decreases in the pulmonary circulation and flow reverses in the left CA, the left CA fails to supply the myocardium and drains oxygenated blood into the main PA. Myocardial ischemia results from the coronary steal phenomenon, in which a left to right shunt leads to decreased LV perfusion [1]. In the present case, an old MI was incidentally detected by echocardiography during the preoperative evaluation for non-cardiac surgery. Because CAG did not show atherosclerotic obstructive coronary stenosis, the decreased myocardial perfusion by ALCAPA was likely the cause of the old MI in this patient.

The treatment of choice for ALCAPA is corrective surgery [1]. Because continuous left to right shunting may increase LV infarct size, thus promoting the development of heart failure or sudden cardiac death, surgical correction of ALCAPA was recommended for this patient. However, the patient refused surgery and selected medical therapy because of the lack of symptoms. Guideline-derived medical therapy for asymptomatic LV dysfunction, including a beta-blocker and angiotensin converting enzyme inhibitor was initiated, and the patient has been asymptomatic without any clinical events over a 2-year clinical follow-up.

Double RCA is also very rare, with an incidence of $0.01 \%$ in the era of CAG and $0.07 \%$ in patients who underwent cardiac CT [4]. In the present case, double RCA was also detected on CT CAG and conventional CAG. This is the first case of a coronary artery anomaly with both adult ALCAPA and double RCA complicated by a silent MI.

Double RCA is known to be a benign disease with no hemodynamic significance, but some authors have suggested that it may be associated with increased atherosclerotic changes in coronary arteries resulting in myocardial ischemia and angina [4]. Therefore, medical therapy and continuous surveillance for atherosclerotic changes in symptomatic or asymptomatic patients are recommended for patients with double RCA. Double RCA with two separate ostia was diagnosed by CT CAG before cardiac catheterization in the present case. Double RCA with two separate ostia may be clinically important when coronary artery intervention for atherosclerotic disease is considered. The coronary intervention may only be performed for one RCA, and not the 
other, which may result in incomplete interventional procedure in the absence of pre-interventional knowledge of double RCA with two separate ostia.

In conclusion, we report an extremely rare asymptomatic case of an old MI, presumably caused by adult type ALCAPA, and double RCA was incidentally diagnosed during $\mathrm{CT}$ and conventional CAG. Coronary anomalies were suspected during the echocardiographic examination. Therefore, physicians should keep in mind that an MI may develop from non-atherosclerotic causes, as in the present case, and multi-modality non-invasive cardiac imaging can be helpful when coronary anomalies are suspected.

중심 단어: 관상동맥 기형; 심근 경색증; 폐동맥

\section{REFERENCES}

1. Peña E, Nguyen ET, Merchant N, Dennie C. ALCAPA syndrome: not just a pediatric disease. Radiographics 2009;29: 553-565.

2. Yau JM, Singh R, Halpern EJ, Fischman D. Anomalous origin of the left coronary artery from the pulmonary artery in adults: a comprehensive review of 151 adult cases and a new diagnosis in a 53-year-old woman. Clin Cardiol 2011;34:204-210.

3. Erbagci H, Davutoglu V, Turkmen S, Kizilkan N, Gumusburun E. Double right coronary artery: review of literature. Int $\mathrm{J}$ Cardiovasc Imaging 2006;22:9-11.

4. Chien TM, Chen CW, Chen HM, Lee CS, Lin CC, Chen YF. Double right coronary artery and its clinical implications. Cardiol Young 2014;24:5-12.

5. von Ziegler F, Pilla M, McMullan L, et al. Visualization of anomalous origin and course of coronary arteries in 748 consecutive symptomatic patients by 64-slice computed tomography angiography. BMC Cardiovasc Disord 2009;9:54.

6. Erol C, Seker M. Coronary artery anomalies: the prevalence of origination, course, and termination anomalies of coronary arteries detected by 64-detector computed tomography coronary angiography. J Comput Assist Tomogr 2011;35:618-624

7. Yamanaka O, Hobbs RE. Coronary artery anomalies in 126,595 patients undergoing coronary arteriography. Cathet Cardiovasc Diagn 1990;21:28-40.

8. Garg N, Tewari S, Kapoor A, Gupta DK, Sinha N. Primary congenital anomalies of the coronary arteries: a coronary: arteriographic study. Int J Cardiol 2000;74:39-46.

9. Attili A, Hensley AK, Jones FD, Grabham J, DiSessa TG. Echocardiography and coronary $\mathrm{CT}$ angiography imaging of variations in coronary anatomy and coronary abnormalities in athletic children: detection of coronary abnormalities that create a risk for sudden death. Echocardiography 2013;30: 225-233. 